\title{
Construction of the Scattering Wave Function through the Analytical Continuation of a Bound State.
}

\author{
N. Milani \\ Istituto di frisica dell'Università - Padova \\ T. A. Minelli and A. Pascolini \\ Istituto di Fisica dell'Università - Padova \\ Istituto Nazionale di Fisica Nucleare - Sezione di Padova \\ (Lett. Nuovo Cimento, 19, 509 (1977))
}

Table I on page 512 is wrongly printed, it should read

TABLE I. - The energies $E_{i}$ of the p-wave bound and quasi-stationary states of the potential (11) as computed in the exact case and with the various approximations $P^{i}$.

\begin{tabular}{lllll}
\hline & Exact solution & $P^{1}(r)$ & $P^{2}(r)$ & $P^{3}(r)$ \\
\hline$\chi^{2}$ & - & $7.28 \cdot 10^{-1} i$ & $6.2 \cdot 10^{-6}$ & $6.15 \cdot 10^{-4}$ \\
\hline$E_{1}$ & -25.7775 & -25.7775 & -25.7785 & -25.8665 \\
\hline$E_{2}-17.6425$ & -17.6425 & -17.6290 & -17.7605 \\
\hline$E_{3}-5.9755$ & -5.9755 & -5.9545 & -6.105 \\
\hline$E_{4}$ & $8.3736-3.1349 i$ & $8.3736-3.1349 i$ & $8.0798-3.3589 i$ & $7.6843-3.3675 i$ \\
\hline$E_{5}$ & $30.3570-6.9280 i$ & $30.3570-6.9280 i$ & $29.5287-7.8637 i$ & $28.5935-8.1055 i$ \\
\hline$E_{6}$ & $57.3047-10.6052 i$ & $57.3047-10.6052 i$ & $54.9538-12.5951 i$ & $52.7641-12.6113 i$ \\
\hline$E_{7}$ & $89.2050-14.4201 i$ & $89.2050-14.4201 i$ & $83.615-16.754 i$ & $80.5500-15.1226 i$ \\
\hline
\end{tabular}

(C) by Società Italiana di Fisica

Proprieta letteraria riservata

Direttore responsablle: CARLO CASTAGNOLI

Stampato in Bologna dalla Tipografia Compositori coi tipi della Tipografla Monograf Questo fascicolo è stato licenziato dai torchi 11 27-IX-1977 